\title{
Autonomous Initiation of Human Physical Assistance by a Humanoid
}

\author{
Anastasia Bolotnikova $^{1,2}$, Sébastien Courtois ${ }^{1}$, Abderrahmane Kheddar ${ }^{2}$
}

\begin{abstract}
We study the use of humanoid robot technology for physical assistance in motion for a frail person. A careful design of a whole-body controller for a humanoid robot needs to be developed in order to ensure efficient, intuitive and secure interaction between humanoid-assistant and human-patient. Here, we present a design and implementation of a wholebody controller that enables a humanoid robot with a mobile base to autonomously reach a person, perform audiovisual communication of intent, and establish several physical contacts for initiating physical assistance. Our controller uses (i) visual human perception as a feedback for navigation and (ii) joint residual signal based contact detection for closed-loop physical contact creation. We assess the developed controller on a healthy subject and report on the experiments achieved and the results.
\end{abstract}

\section{INTRODUCTION}

According to the most recent predictions, by the year of 2050,1 out of every 6 people in the world will be over the age of 65 , compared to 1 out of every 11 for the same age group in 2019 [1], [2]. As a result, demand for healthcare professionals, who can help frail elderly with everyday routine tasks, is expected to increase dramatically. With a shortage of people of working age, who could satisfy this demand, robotization of the caregiving operating industry is required. Moreover, the recent Covid19 outbreak brought to light the crucial role that robots can play in future pandemics.

Robots are expected to play a pivotal role in the future of healthcare for elderly and disabled, improving both quality of life of patients and quality of work of human caregivers [3]. Particularly, user-friendly humanoid robots [4] may be a good fit for the job thanks to their familiar shape [5], potential multi-functionality and natural communication capabilities of a social robot specifically designed to interact with people in a natural, intuitive and useful way [6].

In this work, we consider a general interaction scenario which may occur in everyday care. A humanoid robot is required to autonomously reach a person and establish several physical contacts on a human body to initiate physical assistance in motion. A whole-body quadratic programming (QP) controller framework 1 is used to develop a Finite State Machine (FSM) controller that enables a humanoid robot to autonomously participate in such interaction scenario.

A visual human perception is used for closed loop navigation towards a human. The joint residual signal based contact detection is used to determine time of robot-human contact events [7], [8]. Verbal, visual and body language

\footnotetext{
${ }^{1}$ SoftBank Robotics Europe, Paris, France

${ }^{2}$ University of Montpellier-CNRS LIRMM, Interactive Digital Humans, Montpellier, France

${ }^{1}$ https://jrl-umi3218.github.io/mc_rtc
}

communication is included in the controller design to enable a robot to autonomously communicate its intentions to a human. More specifically, our contributions are following:

- the design of a whole-body controller architecture for the interaction scenario (Sec. IIII-A);

- the implementation of a visual feedback based navigation towards a human (Sec. III-B);

- the integration of a multi-modal communication of intent in the controller design (Sec. [III-C);

- finally, we present the results of using the controller for an interaction trial with a healthy subject (Sec. IV].

\section{BACKGROUND}

In the field of research on physical human-robot interaction (pHRI) for assistance in motion or power augmentation, a large majority of the works consider either the scenarios where human is creating a contact on the robot body surface [9], [10] or the application of exoskeletons [11]. In our work, however, the roles are reversed, it is the robot who is responsible for autonomously establishing a physical contact. An interaction scenario where the robot itself is actively and autonomously creating contacts on a surface of a human participant body is rarely considered. In [12] a control of a robot establishing a contact on the human body was studied with consideration of safety limits in terms of the human pain tolerance limits. In [13] a humanoid robot was used in a sit-to-stand assistance context to contact a human and perform a motion while maintaining the contact.

Human-aware navigation is a basic skill that a robot must have when operating in the same environment as humans [14]. A sensor fusion navigation approach was developed and tested in [15]. More often human avoidance strategies in navigation are studied [16], [17]. Visual servoing was used to make a robot navigate towards a human, maintain constant distance between itself and a human, and follow a walking human. This visual servoing based control was presented in [18], [19]. Although, this work does not include the details of the navigation method. However, a demo of this navigation approach is available online ${ }^{2}$

Yet, none of the previous works have considered a full autonomous interaction scenario with integration of all components of visual feedback based navigation towards a person, multi-modal communication of intent and closed-loop physical contact creation. In our work, we describe the design and implementation of the whole-body QP FSM controller for the studied pHRI scenario (Sec. III) and present the experimental results (Sec. IV).

https://youtu.be/QDmDY5koKIE 


\section{CONTROller ARCHitecture}

The whole-body controller is developed using mc_rtc QP task-space control framework. We consider a complex interaction scenario which is comprised of several distinct stages, each of which consists in achieving multiple objectives. Therefore, the controller is implemented in a form of an FSM, where every state corresponds to a specific stage of the interaction process. Fig. 1] presents the general structure of the implemented FSM controller. In Sec. III-A we describe this controller implementation in detail.

\section{A. FSM QP controller implementation}

The robot actions are controlled by an accelerationresolved QP controller. This means that an optimization problem is formulated with a quadratic objective function that consists of a weighted sum of tasks formulated as errors between actual and desired setpoints in task space, as well as first and second derivatives of these task errors. Robot joints acceleration are the decision variables of the problem. A set of linear constraints in the optimization problem formulation ensure that the solution is physically feasible and safe [20].

As indicated in Fig. 1, the interaction starts with controller initialization (init). At this stage, the robot description module is used to build the base control problem as QP with default tasks and constraints (Eq. 11.

$$
\min _{\ddot{\boldsymbol{q}}, \boldsymbol{f}} \mathcal{P}+\mathcal{B}+\mathcal{C}
$$

s. t. $\left\{\begin{array}{l}\text { joint position, velocity and torque limits } \\ \text { self-collision avoidance } \\ \text { sliding ground contact constraints } \\ \text { bound mobile base velocity and acceleration }\end{array}\right.$

The objective function of the QP contains a default posture task $\mathcal{P}$, default mobile base position task $\mathcal{B}$ and a center of mass task $\mathcal{C}$. Interested reader can refer to [20] for detailed definition of these common QP tasks and constraints.

After the controller initialization, transition to InitialPosture state is triggered. At this stage, we assume that the robot is positioned far from and facing the human participant. In this state, the default posture task $\mathcal{P}$ is given a new setpoint $q_{d}$, which is an upright standing posture. We define a threshold $\delta_{\mathcal{P}}$ and consider this state to be completed when the posture task error $\epsilon_{\mathcal{P}}$ is less than or equal to this threshold, which triggers transition to the NavigateToHuman state.

In the NavigateToHuman state, default mobile base task $\mathcal{B}$ is removed from the problem formulation and a Position Based Visual Servoing (PBVS) task $\mathcal{V}$, regulating mobile base motion, is added. The objective of this task is to minimize the error in the camera frame between mobile base position, computed from kinematics tree, and target mobile base position, which is defined w.r.t human frame detected in robot camera field of view (Fig. 2). We detail how this target position is computed and reached in closed-loop in Sec. IIIB Termination of this state is triggered when $\mathcal{V}$ task error $\epsilon_{\mathcal{B}}$ reaches a predefined threshold $\delta_{\mathcal{B}}$.

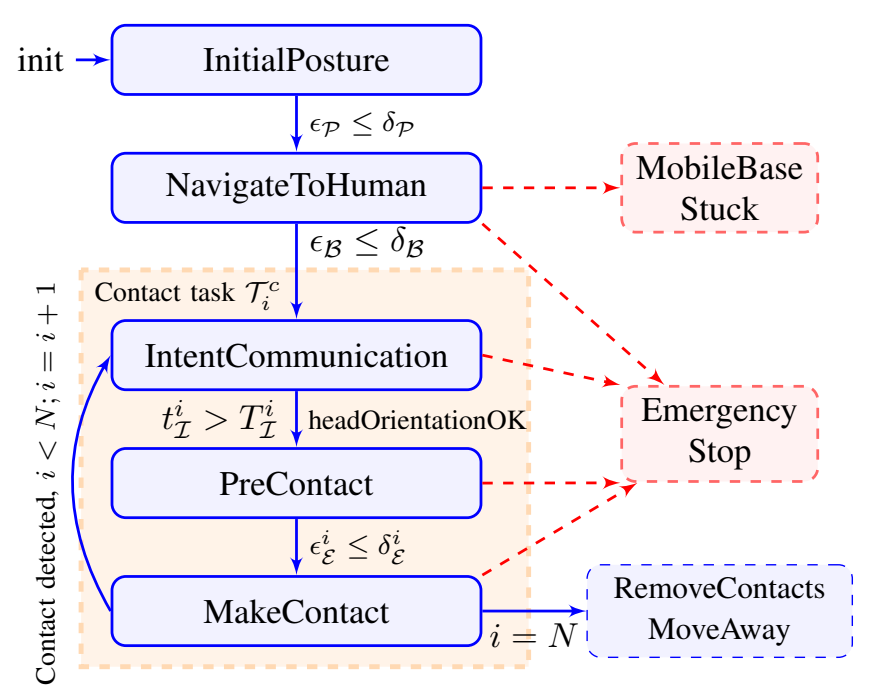

Fig. 1: FSM based controller design for interaction scenario.

Now, the robot executes a sequence of predefined contact tasks $\mathcal{T}_{i}^{c}, i=0, \ldots, N$. For every contact task, the first state (of the sub-FSM) is IntentCommunication, where the robot explains and illustrates its further intentions specific to $\mathcal{T}_{i}^{c}$ to the human (Fig. 3). This state is implemented to ensure smooth and comprehensive transition to the states where the robot establishes physical contacts with the human. We detail the purpose and implementation of this state in Sec. III-C. Human head orientation is monitored in this state to verify that a human has paid attention to the visual communication on the robot screen. The time for intent communication is predefined $T_{\mathcal{I}}$. Once state time $t_{\mathcal{I}}$ exceeds $T_{\mathcal{I}}$ intent is considered to be successfully communicated and transition to the PreContact state is triggered. After robot prepares for a contact, appropriate joint residual signal [7], [8] is monitored in the MakeContact state, as robot is moving its end-effector towards the human body, to detect the time of the contact event. When all contact tasks are finished the experiment ends with RemoveContacts state and moving away.

\section{B. Nearby surrounding navigation towards human}

We define a fixed mobile base position and orientation target w.r.t human reference frame ${ }^{h} X_{b}^{*}$. The human reference frame (Pelvis link) pose in the robot camera frame is obtained from human Body Tracking by Azure Kinect camera installed on the robot, we denote this transformation as ${ }^{c} X_{h}$. From the joint encoder readings and known robot kinematics we compute transformation between camera frame and mobile base frame ${ }^{b} X_{c}$, which at the start of the experiment is equal to the world frame ${ }^{b} X_{c}={ }^{w} X_{c}$ at $t=0$, where $t$ is the time elapsed from the start of the experiment. The desired setpoint pose ${ }^{h} X_{b}^{*}$ in the camera frame is (Eq.22

$$
{ }^{c} X_{b}^{*}={ }^{c} X_{h}{ }^{h} X_{b}^{*}
$$

All frames involved in these computations are illustrated on the experiment simulation setup in Fig. 2 


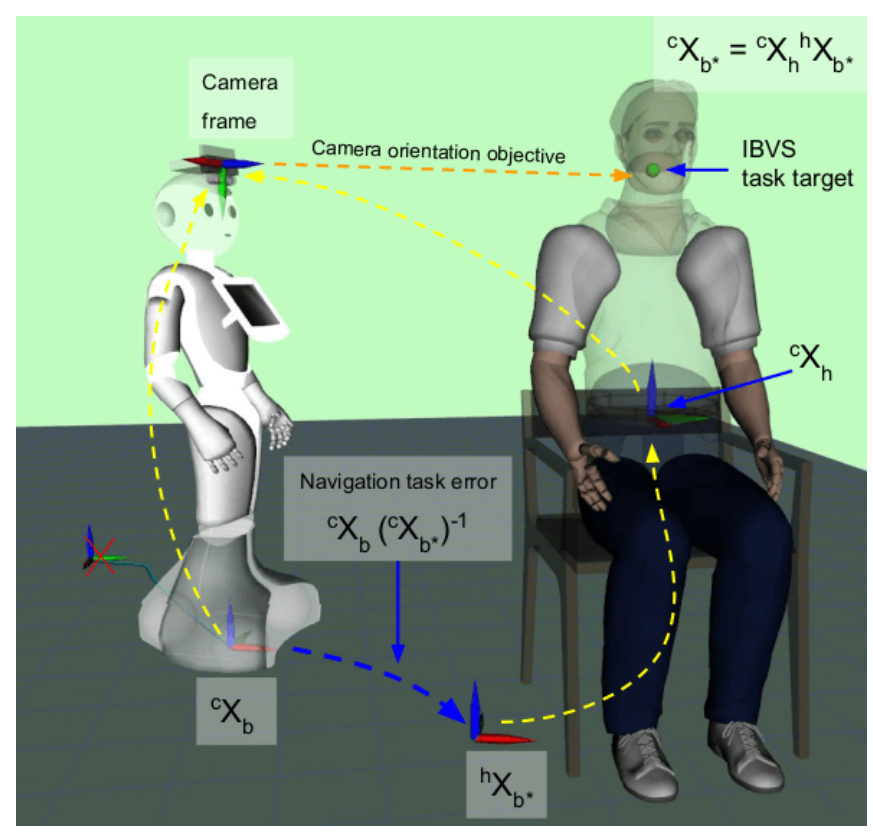

Fig. 2: NavigateToHuman FSM state visual tasks

This update is performed on every iteration of the controller and passed as a target to the PBVS QP task $\mathcal{V}$ associated with the motion of the mobile base. Due to the fact that a camera and the body tracking frame rate $(30 \mathrm{~Hz}$ and $15 \mathrm{~Hz}$ respectively) is much lower than the control frame rate $(83 \mathrm{~Hz}$ in case of Pepper), new detection data is not available for every controller iteration. This may result in a sudden task error jumps and discontinuities. For a smoother PBVS task error evolution and convergence, in this work, we set lower limits for the mobile base speed and acceleration.

The error between the current and desired mobile base frame poses is used as a feedback error for the PBVS task $\mathcal{V}$ to navigate in closed-loop to the desired position (Eq. 3]

$$
\epsilon_{\mathcal{B}}={ }^{c} X_{b}\left({ }^{c} X_{b^{*}}\right)^{-1}
$$

Detected human head frame is used for a camera orientation target task implemented in the controller as an Image Based Visual Servoing (IBVS) task $\mathcal{O}$. With head $X_{c}$ being a human head frame pose in the camera frame detected by Azure Kinect, the $\mathcal{O}$ task error which ensures that human head is kept as close as possible to the center of the field of view, $(\mathrm{FoV})$ is $\epsilon_{\mathcal{O}}={ }^{\text {head }} X_{c}$.translation Based on our experience, keeping a human head in the center of FoV results in a better overall human body tracking results from the Azure Kinect, especially once some human body parts become occluded and are thus no longer in the FoV.

In the future, we consider combining this method with VSLAM $3^{3}$ technology for highly robust performance. This is necessary for improved safety and for handling low frame rate and high frame latency of Azure Kinect depth sensor.

\footnotetext{
${ }^{3}$ We choose SLAM as it will certainly be a mature technology to navigate inside rooms indoor senior citizens' homes, personal houses, hospitals, etc.
}

\section{Intent communication for user comfort and safety}

Humans usually express interest in touching a humanoid robot when they see one. However, when the roles are reversed and it is the robot that will establish physical contacts on a human body, the loss of control from a human side, the lack of understanding of the robot intentions, and the low communicative cognitive capabilities of the robot can cause discomfort and even fear during the interaction. The closer a robot is to a person, the more considerations need to be taken into account for human comfort and safety. And in the case of direct physical contact, human participant safety and comfort (both physical and mental) are of extreme importance for successful human-robot interaction.

Indeed, a human participant needs to trust the robot to feel comfortable to allow it to establish physical contact. And this trust can be established only if the human can predict what the robot intentions are. Therefore, we integrate good user experience design considerations as parts of the FSM controller in order for the human to feel comfortable while the robot is in close proximity and is establishing physical contacts with the human. For that robot is programmed to clearly communicate its intentions to the user, using different communication modalities (verbal, visual, body language).

When the robot reaches the person close enough, prior to establishing physical contacts, the robot is communicating verbally what it intends to do next. At the same time, using body language the robot draws the attention of the human participant to its tablet screen where an illustration of the intended physical interaction is displayed on a schematic figure, as is shown in Fig. 3 .

With these familiar tools for information transfer, we enrich the interaction with different communication modalities and enable the robot to make its intentions very clear prior to establishing physical contact.

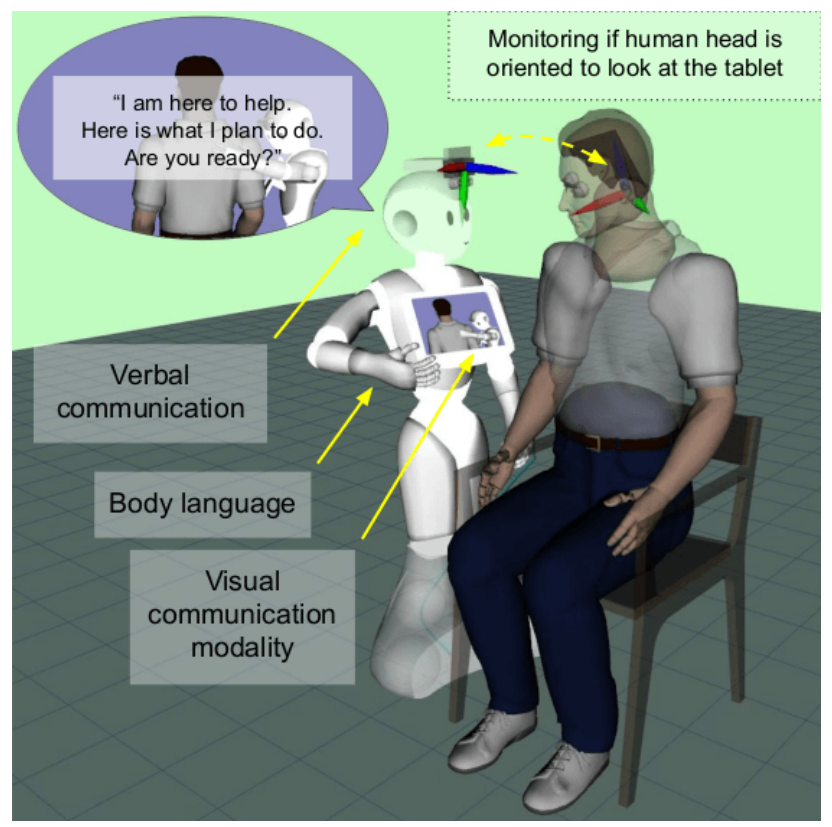

Fig. 3: Multi-modal IntentCommunication FSM state 


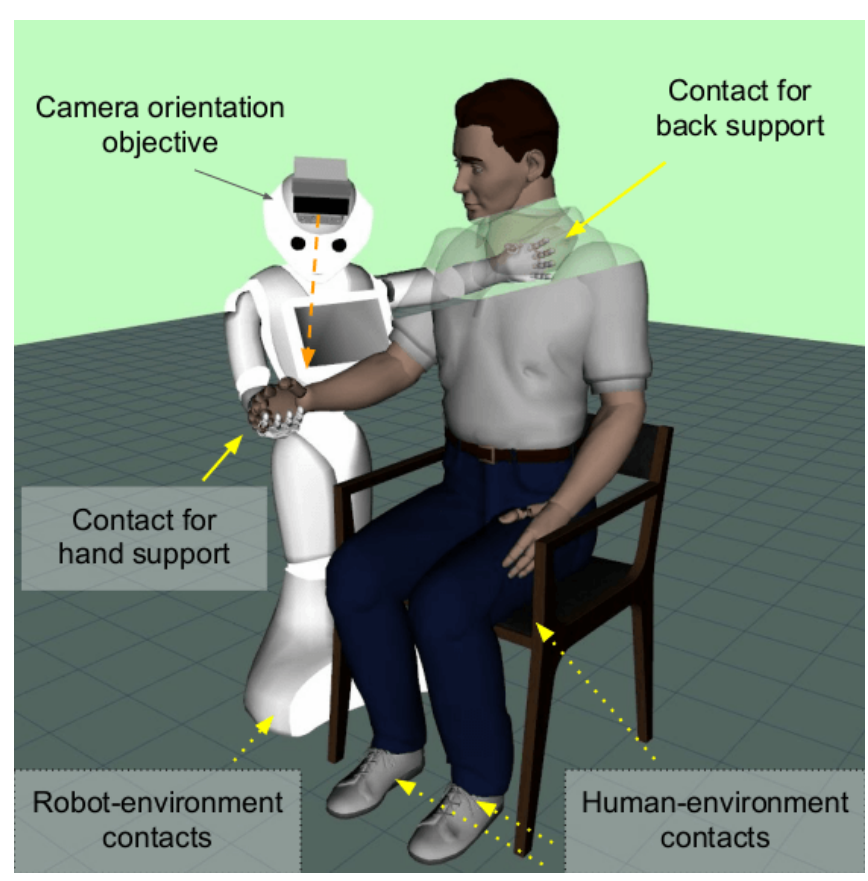

Fig. 4: MakeContact FSM state, all contacts established

\section{Physical contact establishment}

The physical contacts are established using posture QP tasks to move robot's right or left hand links towards the surface of the uman body where the contact is supposed to be established (Fig. 4). Contacts are established in closedloop via monitoring of residual signal between predicted (learned) and measured joint position tracking errors [7], [8]. Predefined contact locations on a human body in this work were inspired from observing the human caregiver's practices for similar interaction tasks. The exact position of the contacts can be adjusted to different human morphologies or chair heights. However, the feasible contact locations are limited by the robot's reachable workspace. The exact feasible placement of the contacts can be planned by analyzing the human point cloud using the method presented recently [21].

\section{EXPERIMENTAL RESULTS}

We have achieved preliminary experiments that will be enhanced further in the future work 4

\section{A. Platform description}

We are using the upgraded prototype of Pepper wheeled humanoid robot platform that has been customized for later in-situ experiments with real patients. With respect to the commercial version, this robot has an additional Azure Kinect camera mounted on the top of the head, mainly for human body tracking. The robot is also equipped with the RealSense D434 camera for SLAM and additional source of IMU measurement that we are not using in this paper. These additional hardware elements are added to the robot description module and used by mc_rtc framework to

\footnotetext{
${ }^{4}$ For the time being, the experiments with more participants couldn't be achieved because of the restrictions of the Covid19 outbreak.
}

build the base controller QP formulation. Under the tablet, this Pepper version has embedded Jetson TX2 for image processing and other heavy computation. For the time being, we are running the controller on an external PC for easy debugging and programming purposes.

\section{B. Results}

At the start of the preliminary experiment, the robot is placed about 1.2 meters away from a chair where the human participant is sitting. First, the robot navigates towards the person, then communicates its intentions, and proceeds to initiate several physical contacts. First, robot establishes a contact with the right shoulder of the human participant. Then, the robot invites the participant to place their hand in the robot's right end-effector (as is being simultaneously demonstrated on the tablet screen of the robot). The experiment ends with all contacts being autonomously removed, robot thanking the participant and moving away.

Fig. 6 shows main stages of the experiment. Interested reader is invited to see the video accompanying this paper for the full experiment demonstration or to refer to the controller source code for implementation details 5

Plot on Fig. 5 shows evolution of the PBVS task errors in $\mathrm{XY}$ translational and $\mathrm{Z}$ rotational axes during the NavigateToHuman state (Fig. 6a). The task error evolution and convergence is mostly smooth. A slight sudden jump occurs at the end of the state for the $Y$ axis, when the robot is very close to the human participant. Typically, at this stage the depth perception deteriorates due to the human being too close to the camera and, as a result, discontinuities in human body tracking are also likely to occur. Nevertheless, the NavigateToHuman state completion criteria is successfully reached and transition to the next FSM state is triggered.

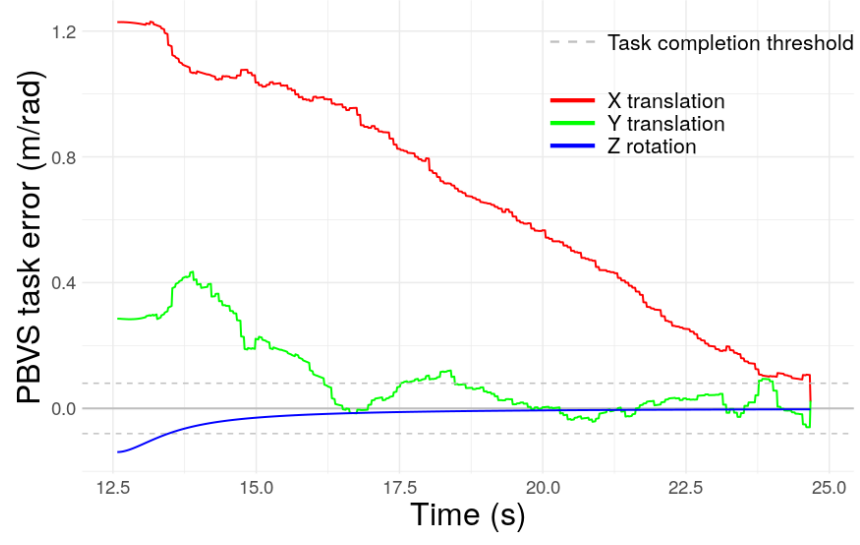

Fig. 5: Evolution of navigation task errors

Next state in the FSM is IntentCommunication. This part of the experiment can be seen on Fig. 6b. While the robot is communicating its intentions, using multiple communication modalities, as described in Sec. III-C, the IBVS task is responsible for keeping the human head in the field of view.

https://github.com/anastasiabolotnikova/autonomous phri_init 

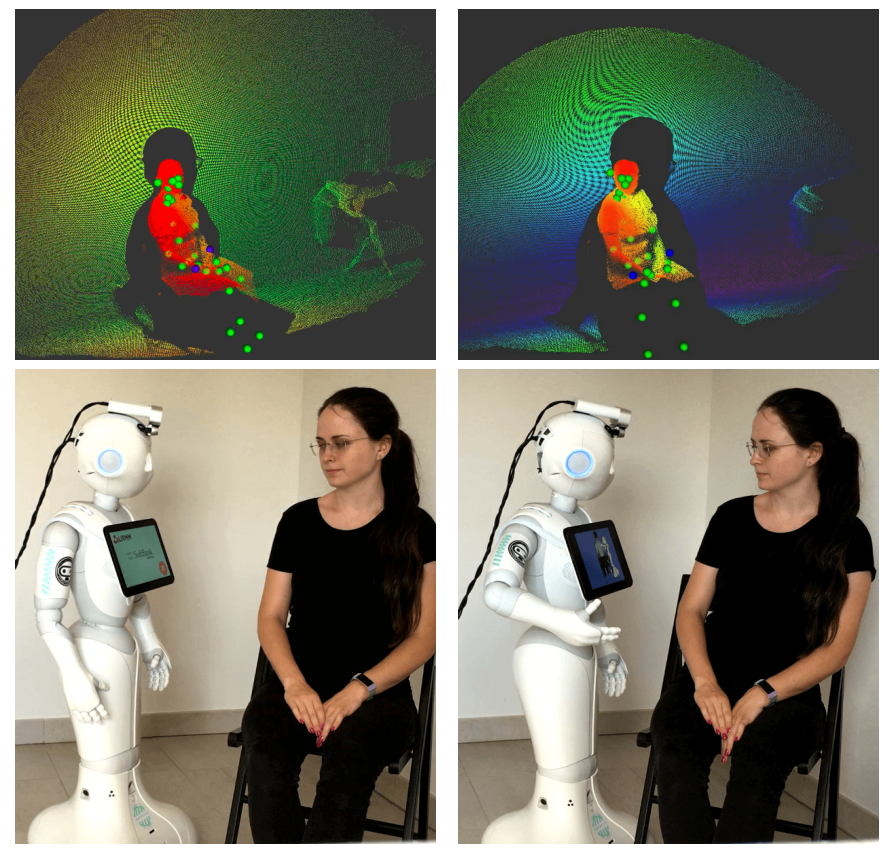

(a) Navigation

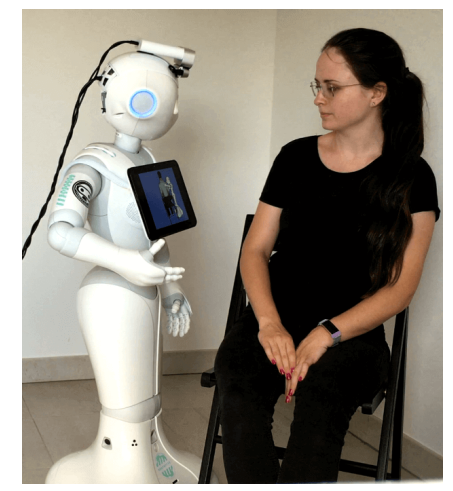

(b) Intent communication
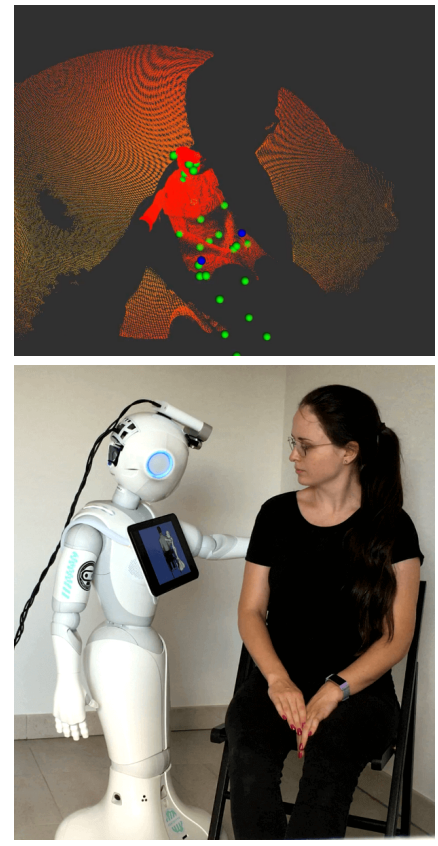

(c) First contact
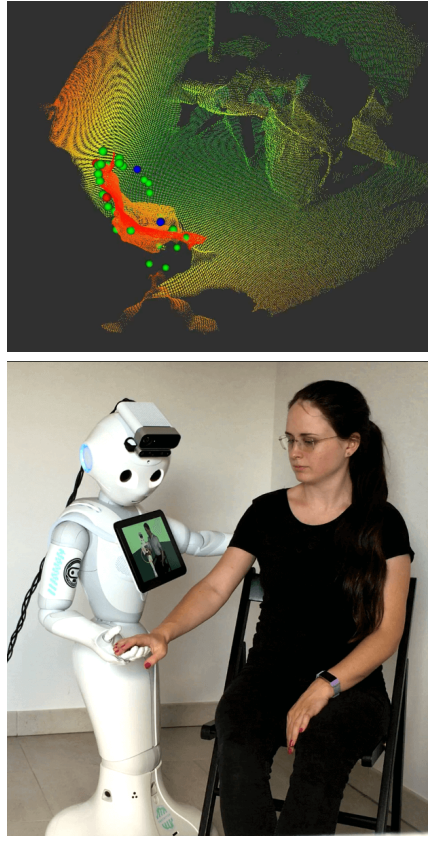

(d) Second contact

Fig. 6: Robot onboard camera view and video screenshots illustrating main parts of the experiment with human participant

The orientation of the human head frame is monitored, and the robot checks if the human face was oriented to look at the robot's tablet at least once for paying attention to the figure displayed on the screen. This is an additional criteria for exiting IntentCommunication state. The plot of the monitored human head to robot tablet angle is shown on Fig.7 This plot validates that, shortly after the start of IntentCommunication state, the human head is being oriented to look at the tablet.

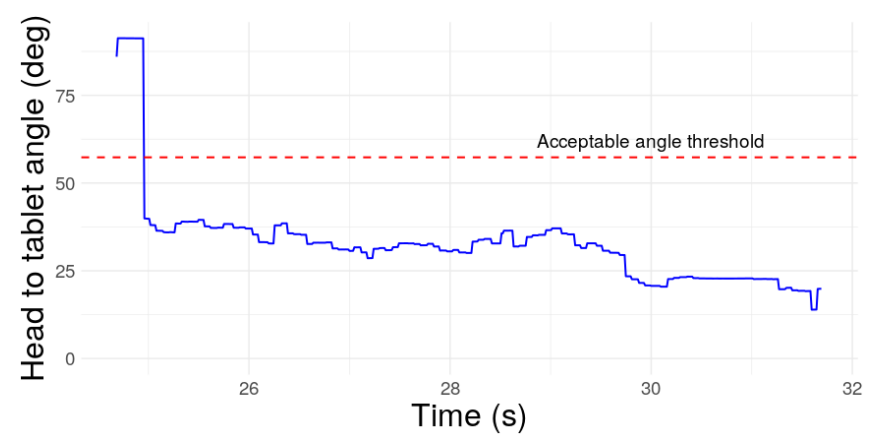

Fig. 7: Human face to tablet angle

Once intent is communicated, the transition to PreContact and then MakeContact states for the robot's left end-effector are triggered. The robot starts to move its left arm towards human back to establishes a physical contact on the right shoulder of the participant (Fig. 6c). The monitoring of the residual between measured left shoulder joint position tracking error and predicted expected position tracking error of this joint allows to detect the contact event (as mentioned in Sec. [II-D). Fig. 8 shows the residual signal for the left robot shoulder roll joint. As can be seen from the plot, the contact is detected (when residual reached a threshold) and maintained for several seconds after the detection occurs as is requested according to the MakeContact state design.

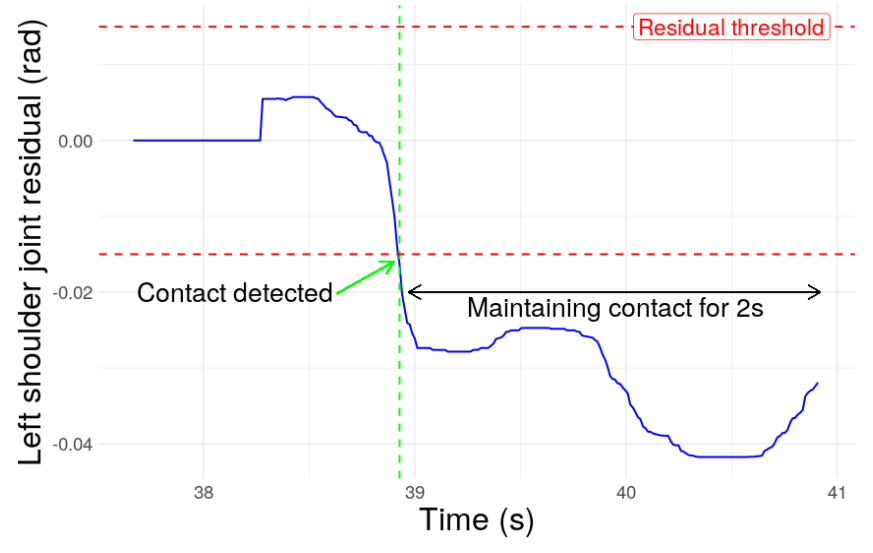

Fig. 8: Left end-effector contact detection

Similar process is repeated for the robot's right endeffector. However, in this case, the robot invites the user participant to place their hand into the robot's right end effector, which is brought up in front of the user as is shown in (Fig. 6d). The plot on the Fig. 9 shows the residual signal for the robot right elbow roll joint and indicates successful detection of the contact event. Once the contact is detected, the robot closes its right gripper slightly, as a human would do in a similar interaction. This contact is also maintained for a few seconds before being removed.

The experiment ends with the robot autonomously and carefully removing all established physical contact one by one. The robot thanks the human user for participation in the experiment and moves away. 


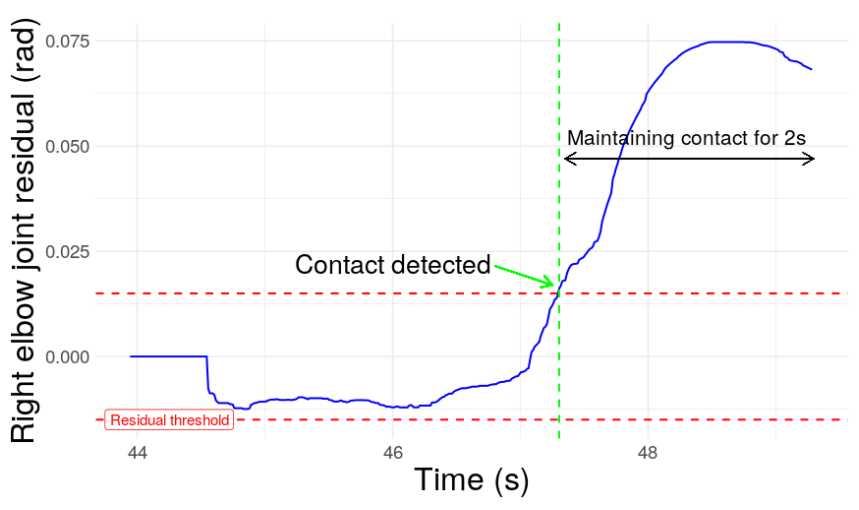

Fig. 9: Right end-effector contact detection

\section{CONCLUSION AND FUTURE WORK}

In this paper, we integrated and enhanced different bricks to build an architecture that enables the humanoid robot Pepper to reach a person in need for physical assistance, communicate its intentions and establish several physical contacts to initiate the assistance process. We developed a taskspace optimization controller instance derived from [20], enhanced with visual servoing [22], which allows reaching safely a person. In close contact interaction the robot initiates contacts [7] to achieve compliant contact motion [8]. The controller was enhanced with communication plug-ins.

We believe that all the bricks that have been developed are viable ground technologies to effectively achieve frail pHRI purpose in real experiments. The latter are planned to be conducted in-situ a retirement house with few real patients in our future work; where participant impression during the interaction will also be evaluated. Engineering efforts are now undertaken to clean the existing software to meet safety requirements and recovery procedures. SLAM technology will certainly mature to a real-time reliable and commercially available software and is integrated (but not used in this paper) to navigate in indoor spaces. We forecast the same maturation for human body tracking. Task-space optimization have been proved extremely flexible and reliable in many industrial set-up. In near future work, the human model will be integrated as part of the multi-robot QP control model [20]. Thereby, we are synthesizing an adaptive force controller in the task space to allow the robot to effectively assist the site-to-stand motion, human limbs manipulation, etc. The latter problem is challenging because a human is not equipped with encoders or torque sensing which would allow closing the loop and computing the right amount of assistive forces. We will also redesign the balance criteria for the humanoid to increase safety of the dyad during pHRI.

\section{REFERENCES}

[1] "World population prospects 2019. highlights," report ST/ESA/SER.A/423, United Nations, Department of Economic and Social Affairs, Population Division, New York, US, 2019.

[2] "World population ageing 2019. highlights," report ST/ESA/SER.A/430, United Nations, Department of Economic and Social Affairs, Population Division, New York, US, 2019.
[3] M. Niemelä and H. Melkas, "Robots as social and physical assistants in elderly care," in Human-Centered Digitalization and Services, vol. 19, pp. 177-197, 2019.

[4] A. Pandey and R. Gelin, "A mass-produced sociable humanoid robot: pepper: the first machine of its kind," IEEE Robotics \& Automation Magazine, vol. 25, no. 3, pp. 40-48, 2018.

[5] M. Staffa and S. Rossi, "Recommender interfaces: the more humanlike, the more humans like," in International Conference on Social Robotics, (Kansas City, US), pp. 200-210, 1-3 November 2016.

[6] S. B. Daily, M. T. James, D. Cherry, J. J. P. III, S. S. Darnell, J. Isaac, and T. Roy, "Affective computing: historical foundations, current applications, and future trends," in Emotions and Affect in Human Factors and Human-Computer Interaction, pp. 213-231, Elsevier, 2017.

[7] A. Bolotnikova, S. Courtois, and A. Kheddar, "Contact observer for humanoid robot pepper based on tracking joint position discrepancies," in IEEE International Conference on Robot and Human Interactive Communication, (Nanjing, China), pp. 29-34, 27-31 August 2018.

[8] A. Bolotnikova, S. Courtois, and A. Kheddar, "Compliant robot motion regulated via proprioceptive sensor based contact observer," in IEEERAS International Conference on Humanoid Robots, (Beijing, China), pp. 854-859, 6-9 November 2018

[9] Y. Tirupachuri, G. Nava, L. Rapetti, C. Latella, and D. Pucci, "Trajectory advancement during human-robot collaboration," in 2019 28th IEEE International Conference on Robot and Human Interactive Communication (RO-MAN), (New Delhi, India), pp. 1-8, 14-18 October 2019.

[10] F. Romano, G. Nava, M. Azad, J. Čamernik, S. Dafarra, O. Dermy, C. Latella, M. Lazzaroni, R. Lober, M. Lorenzini, et al., "The codyco project achievements and beyond: Toward human aware whole-body controllers for physical human robot interaction," IEEE Robotics and Automation Letters, vol. 3, no. 1, pp. 516-523, 2017.

[11] L. M. Vaca Benitez, M. Tabie, N. Will, S. Schmidt, M. Jordan, and E. A. Kirchner, "Exoskeleton technology in rehabilitation: Towards an emg-based orthosis system for upper limb neuromotor rehabilitation," Journal of Robotics, 2013.

[12] Y. Yamada, Y. Hirasawa, S. Huang, Y. Umetani, and K. Suita, "Humanrobot contact in the safeguarding space," IEEE/ASME transactions on mechatronics, vol. 2, no. 4, pp. 230-236, 1997.

[13] A. M. López, J. Vaillant, F. Keith, P. Fraisse, and A. Kheddar, "Compliant control of a humanoid robot helping a person stand up from a seated position," in IEEE-RAS International Conference on Humanoid Robots, (Madrid, Spain), pp. 817-822, 18-20 November 2014.

[14] T. Kruse, A. K. Pandey, R. Alami, and A. Kirsch, "Human-aware robot navigation: A survey," Robotics and Autonomous Systems, vol. 61, no. 12, pp. 1726-1743, 2013.

[15] M. Tee Kit Tsun, B. T. Lau, and H. Siswoyo Jo, "An improved indoor robot human-following navigation model using depth camera, active ir marker and proximity sensors fusion," Robotics, vol. 7, no. 1, p. 4, 2018.

[16] C.-P. Lam, C.-T. Chou, K.-H. Chiang, and L.-C. Fu, "Human-centered robot navigation-towards a harmoniously human-robot coexisting environment," IEEE Transactions on Robotics, vol. 27, no. 1, pp. 99$112,2010$.

[17] A. R. Araujo, D. D. Caminhas, and G. A. Pereira, "An architecture for navigation of service robots in human-populated office-like environments," IFAC-PapersOnLine, vol. 48, no. 19, pp. 189-194, 2015.

[18] G. Claudio, F. Spindler, and F. Chaumette, "Vision-based manipulation with the humanoid robot romeo," in IEEE-RAS 16th International Conference on Humanoid Robots (Humanoids), pp. 286-293, 2016.

[19] D. J. Agravante, G. Claudio, F. Spindler, and F. Chaumette, "Visual servoing in an optimization framework for the whole-body control of humanoid robots," IEEE Robotics and Automation Letters, vol. 2, no. 2, pp. 608-615, 2016.

[20] K. Bouyarmane, K. Chappellet, J. Vaillant, and A. Kheddar, "Quadratic programming for multirobot and task-space force control," IEEE Transactions on Robotics, vol. 35, no. 1, pp. 64-77, 2019.

[21] A. Bolotnikova, S. Courtois, and A. Kheddar, "Multi-contact planning on humans for physical assistance by humanoid," IEEE Robotics and Automation Letters, vol. 5, no. 1, pp. 135-142, 2020.

[22] A. Paolillo, K. Chappellet, A. Bolotnikova, and A. Kheddar, "Interlinked visual tracking and robotic manipulation of articulated objects," IEEE Robotics and Automation Letters, vol. 3, pp. 2746-2753, October 2018. 